\title{
Non-equilibrium Computer Simulations of Coupling Effects under Thermal Gradients
}

\author{
Fernando Bresme \\ Department of Chemistry, Imperial College London, SW7 2AZ, United Kingdom \\ and \\ Department of Chemistry, Norwegian University of Science and Technology \\ E-mail:f.bresme@imperial.ac.uk
}

Received: 09 March 2017; revised: 12 April 2017; accepted: 06 May 2017; published online: 30 September 2017

\begin{abstract}
In this work, we discuss recent developments in the computer simulations of molecular fluids under thermal gradients. Non-equilibrium simulations allow performing numerical tests of fundamental questions of non-equilibrium thermodynamics. These tests show that non-equilibrium simulations provide an efficient approach to quantify within a single simulation the thermophysical properties of fluids along an isobar. We discuss aspects connected to the computation of local temperatures in systems under the influence of heat fluxes, and how the combination of non-equilibrium molecular dynamics and non-equilibrium thermodynamics allows understanding phenomena arising from the coupling of internal molecular variables and heat fluxes, which lead, e.g. to thermo-molecular orientation. The behavior of these orientational effects near a fluid critical point is also discussed.
\end{abstract}

Key words: non-equilibrium molecular dynamics, thermal gradients, local equilibrium hypothesis, configurational temperature, thermal polarization

\section{INTRODUCTION}

Thermal gradients induce fascinating non-equilibrium phenomena and coupling effects in fluids. Ludwig [1] and later Soret [2] showed that thermal gradients drive concentration gradients in aqueous solutions (thermodiffusion), with the ions migrating preferentially towards hot or cold regions. Recent studies have expanded the application of thermal gradients to more complex fluids, such as polymers and biomolecules (thermophoresis) [3-6]. Recently, it has been discovered that thermal gradients induce Casimir-like forces between plates maintained at different temperatures [7]. The applicability of thermophoresis in sensing devices has also been noted [8].

Thermodiffusion and thermophoresis effects are very sensitive to the average temperature of the system of interest. Small changes in temperature can induce a drift of the solutes to hot or cold regions. Considerable effort has been devoted to develop predicting theories to explain such behavior. Some theoretical approaches have allowed us to gain a deeper understanding of these thermal transport phenom- ena [9-11]. However, a full predictive theory of thermophoresis and thermodiffusion is still outstanding. In this sense, non-equilibrium computer simulations are of significant interest, as they can predict Soret coefficients, heats of transport or Seebeck coefficients in solutions, using as input state of the art forcefields [12].

In this contribution, I discuss the applicability of nonequilibrium molecular dynamics (NEMD) simulations to study molecular fluids under thermal gradients. NEMD serves as a microscopic platform to investigate and predict the nonequilibrium response of fluids and to address fundamental questions of fluids out of equilibrium, such as local equilibrium, local temperature, or coupling of molecular internal degrees of freedom with heat fluxes.

\section{SIMULATING THERMAL GRADIENTS}

The application of computer simulations to nonequilibrium phenomena has expanded considerably the scope of theoretical studies of thermodiffusion, as well as the com- 


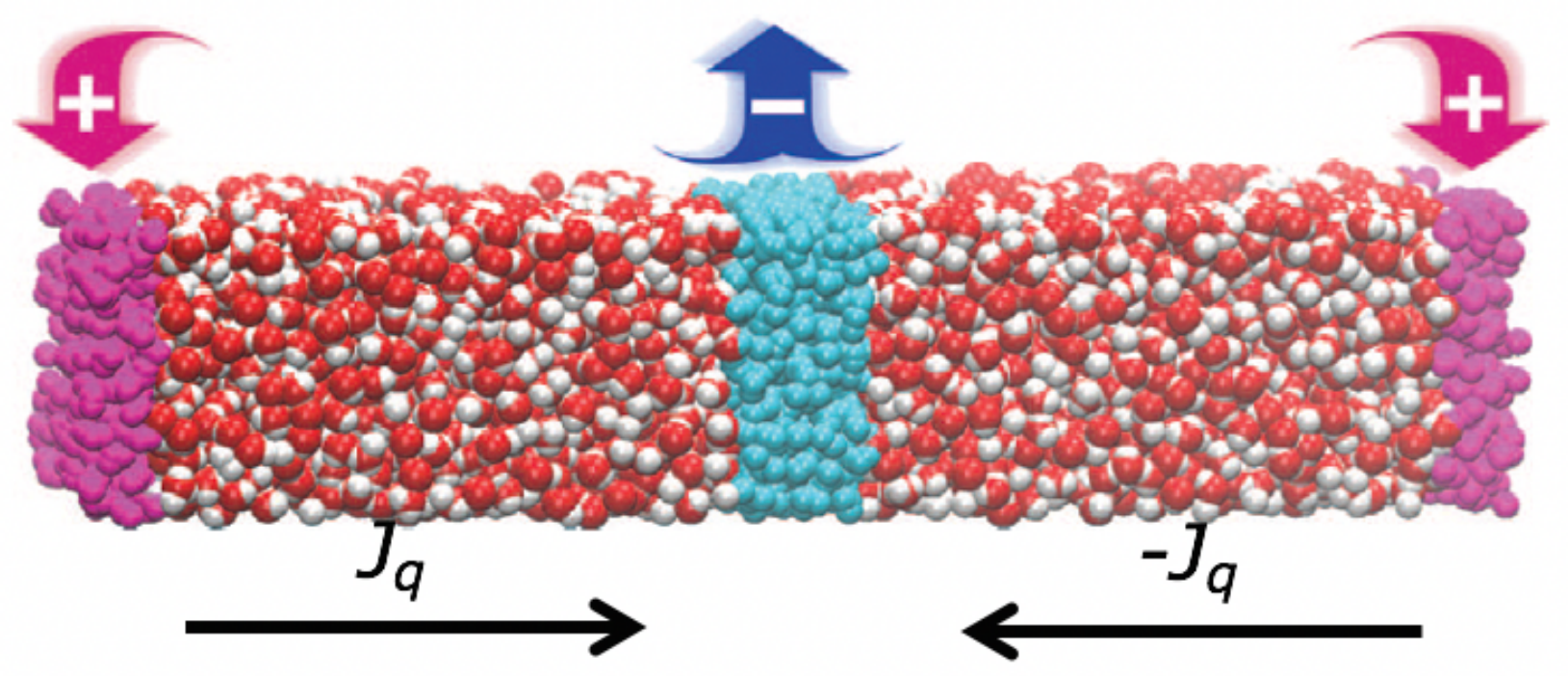

Fig. 1. Simulation set up employed to generate thermal gradients in NEMD simulations. The magenta and cyan regions represent the hot and cold thermostats, respectively. $J_{Q}$ is the heat flux. The fluid consists of water molecules (red - oxygen, white - hydrogen). Figure adapted from reference [18]

putation of thermal transport properties. Non-equilibrium molecular dynamics simulations (NEMD) provide a powerful tool to investigate complex fluids under thermal gradients. NEMD is a microscopic approach that relies on the knowledge of position and momenta of individual particles. Following the seminal work on non-equilibrium simulations in the 1970's by Hoover and Evans [13, 14], several methods have been proposed and improved to simulate systems under thermal gradients (see e.g. ref. [15]). This section focuses on the simulation of explicit thermal gradients as a route to obtain thermal transport properties of fluids. We note that Evans introduced a homogenous method that relies on an extension of the linear response theory, which allows the computation of the thermal conductivity without an explicit temperature gradient [16]. In this approach, the heat flux is generated by applying a fictitious heat field instead of a temperature gradient. Large values of heat fields result in instabilities that are connected to the generation of shock waves. Corrections to address this problem were reported in ref. [17].

Figure 1 shows one possible implementation of a thermal gradient in an NEMD simulation. Distinct boundary regions are introduced in the simulation box to modify the internal energy of the particles and hence to set up the thermal gradient. These boundary conditions are widely used in NEMD simulations [19-22], as they allow the implementation of fully periodic cells. In the approach represented in Figure 1 two thermostats are employed, one in the middle of the simulation cell, where the particles are thermostatted at a temperature $T_{1}$, and another one at the edges of the cell, where the particles are themostatted at temperature $T_{2}>T_{1}$ (see Figure 1). The thermostatting is performed conserving the linear momentum of the system. The coordinates of the particles do not change between thermostatting events and the change in kinetic energy is equal to the change in internal energy. The use of thermostats offers advantages as one can define precisely the temperature range to be investigated in the non-equilibrium simulation. Different thermostats can be employed: simple re-scaling, where the old velocities are rescaled via, $\mathbf{v}_{i, \text { new }}=\chi \mathbf{v}_{i \text {, old }}$, with $\chi=\sqrt{K_{t} / K_{\text {old }}}$ being the ratio between the "old" and the "target" temperatures. More sophisticated canonical thermostats [23] can also be implemented in the simulations [22]. It has been found in a wide range of fluids that the results are fairly independent of the thermostat employed. The method shown in Figure 1 induces two heat fluxes that act in opposite directions. The average heat flux can be obtained from the continuity equation,

$$
J_{Q}=\frac{\langle\Delta U\rangle}{2 \delta t A}
$$

where $\langle\Delta U\rangle$ is the average change of internal energy (kinetic energy) in the thermostatting process, $\delta t$ is the simulation time step and $A$ the cross-sectional area of the simulation box. The heat flux can then be used to compute the thermal conductivities using Fourier's law, $\lambda=-J_{q} / \nabla T$. Additionally, the heat flux can be calculated using the statistical mechanics equation proposed by Irving and Kirkwood (IK) [24]. In most simulations the computations of pressure tensors are performed with a simplified $v s$. of the IK equation, sometime referred to a $I K I$, that is exact for fluids with uniform density. 

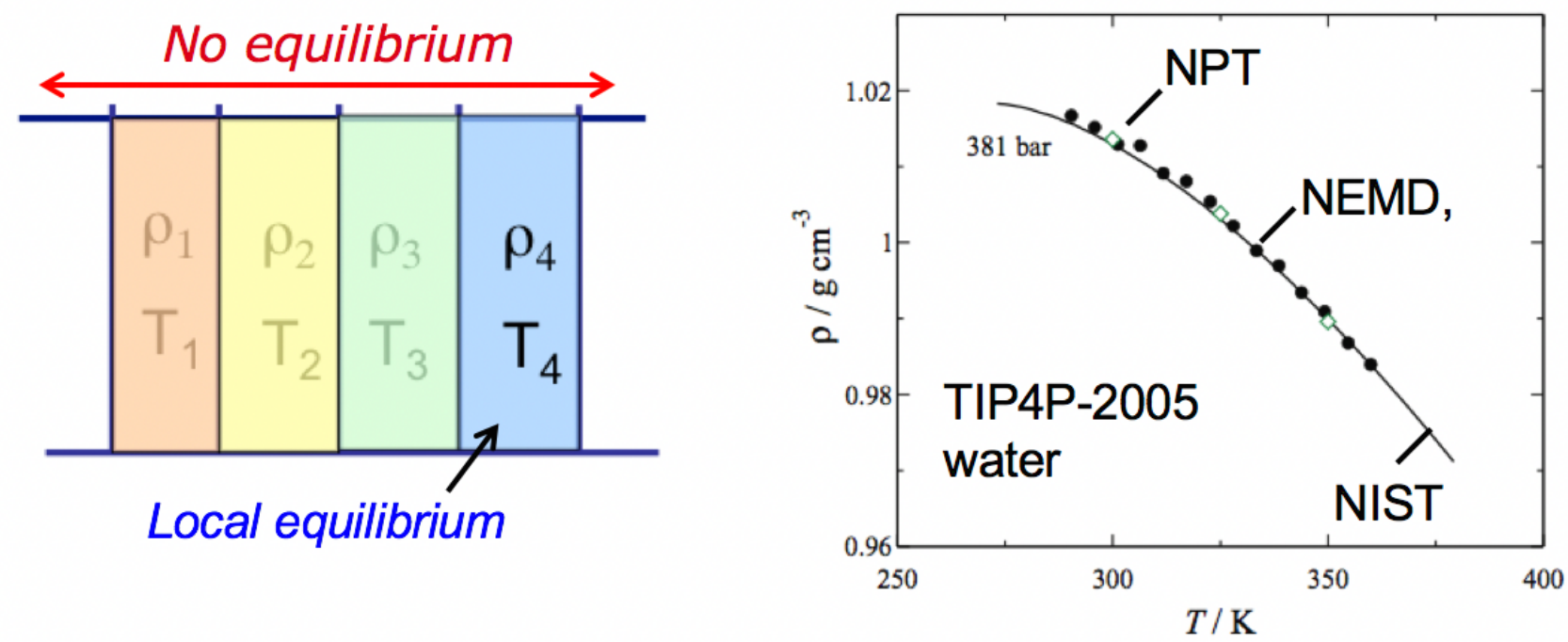

Fig. 2. (Left) Sketch of the states generated in a non-equilibrium simulation, where the thermal gradient induces a density gradient, while the pressure is constant along the simulation cell. The system is under non-equilibrium conditions, but we can define locally the density and temperature, and from this construct an equation of state along an isobar. (Right) Equation of state of TIP4P-2005 water obtained from NEMD and equilibrium (NPT) simulations in the isothermal-isobaric ensemble. The NIST data are included as a reference of the experimental behavior. Figure adapted from reference [29]

For strongly inhomogeneous fluids featuring large changes in density, e.g. fluids next to walls, the IK1 equation becomes inaccurate. In this case the method of planes can be used to calculate the pressure tensor [25]. The approximate equation IK1 has been tested extensively in computations of the heat flux in fluids under thermal gradients, which due to thermal expansion, do also involve density gradients. It has been shown that the IK1 equation predicts heat fluxes in agreement with those calculated from the continuity equation (see refs. [15, 26]).

The thermal gradients employed in NEMD simulations are typically rather large, $\sim 10^{10} \mathrm{~K} / \mathrm{m}$. Such gradients reduce significantly the impact of thermal fluctuations and lead to a good increase of the signal to noise ratio. In fact, the attempt to improve this ratio was one of the key motivations behind the development of non-equilibrium techniques. Early studies of thermal transport properties relied on the use of the equilibrium Green-Kubo approach. The lack of enough statistics resulted in poor signal-to-noise ratios. Despite the large thermal gradients employed in NEMD simulations, it has been shown that the response of the fluids to the thermal gradients is in most cases in the linear regime. This notion can be conveniently tested by calculating the dimensionless number, $\nabla T T^{-1} a$, where $a$ is the characteristic length defining the heat transport. For fluids, $a$ is about one molecular diameter, hence, $\nabla T T^{-1} a<<1$, indicating that the transport is defined mostly in the diffusive regime.

One advantage of the NEMD numerical studies is the possibility of computing local properties, such as density and temperature. This fact makes it possible to calculate the equa- tion of state of a fluid in a single simulation (along an isobar), and to assess any possible deviations of this "non-equilibrium" equation of state from the equilibrium one. This analysis is relevant to address the validity of fundamental principles employed in the Non-Equilibrium Thermodynamics theory, such as the local equilibrium hypothesis (LEH) [28]. This hypothesis states that although the whole system is not at equilibrium, there exists, within a small region, a state of local equilibrium, where the entropy follows from the Gibbs equation, $T d s=d u+p d v-\sum_{i=1}^{n} \mu_{i} d c_{i}$, where $n$ is the number of components in the system of interest. According to this hypothesis, the entropy in the non-equilibrium system could be described in terms of the same thermodynamic properties that apply to the true equilibrium state, namely, the internal energy $(u)$, the specific volume $(v)$, the pressure $(p)$, the chemical potential $\left(\mu_{i}\right)$ and the mass fraction $\left(c_{i}\right)$. Computer simulations provide a route to test numerically this hypothesis, e.g. by comparing the equation of state generated from NEMD with that obtained from equilibrium simulations. Figure 2 shows such a comparison for water, which is modeled in this example using the TIP4P-2005 model. The application of a thermal gradient in the NEMD simulation results in a density gradient, whose magnitude is determined by the thermal expansion of the fluid. One can show that the temperature and density follow the equation of state at equilibrium (See Figure 2), hence supporting numerically, the description of the properties of non-equilibrium systems with equilibrium properties. The TIP4P-2005 model is particularly accurate, as it can be inferred by comparing the simulated results with the experimental ones (c.f. National Institute of 
Standards and Technology database (NIST), NPT and NEMD data in Figure 2).

\section{CALCULATING TEMPERATURES}

NEMD simulations involving thermal gradients require the computation of the temperature. As this is done in a nonequilibrium system, a definition is needed. Such a definition is implicit in the simulations discussed above, for which the equipartition principle was employed. However, the definition of the temperature away from equilibrium has motivated a number of interesting works and discussions. Reference [30] is of particular interest in this context. One important question is whether different definitions of the temperature can provide the same results. Again NEMD allows a numerical test of this question.

The equipartition principle states that,

$$
\frac{1}{k_{B} T_{k i n}}=\left\langle\frac{3 N}{\sum_{i=1}^{N} \frac{\mathbf{p}_{i}^{2}}{m_{i}}}\right\rangle
$$

where $m_{i}$ and $\mathbf{p}_{i}$ are the mass and momentum of particle $i$, $N$ is the total number of particles, and the brackets indicate an ensemble average. In equation (2) we are considering an atomic fluid (hence 3 degrees of freedom per atom). Other definitions for the temperature can be derived using statistical mechanics [31]. Consider a general property, $X\left(\mathbf{r}^{N}\right)$ that is a function of the particle coordinates [32],

$$
\begin{aligned}
\left\langle X\left(\mathbf{r}^{N}\right) \frac{\partial V_{N}}{\partial \xi_{i}}\right\rangle & =\frac{k_{B} T}{Z_{N}} \int \cdots \int \frac{\partial X\left(\mathbf{r}^{N}\right)}{\partial \xi_{i}} \\
& \times \exp \left(-\beta V_{N}\right) d \mathbf{r}_{1} \cdots d \xi_{i} \cdots d \mathbf{r}_{N}
\end{aligned}
$$

where $V_{N}$ represents the total potential energy for $N$ particles, and $\xi_{i}$ are the cartesian coordinates of particle $i$. From equation (3) it follows,

$$
\left\langle X\left(\mathbf{r}^{N}\right) \frac{\partial V_{N}}{\partial \xi_{i}}\right\rangle=k_{B} T\left\langle\frac{\partial X\left(\mathbf{r}^{N}\right)}{\partial \xi_{i}}\right\rangle
$$

which is the Yvon's theorem. [32]. Applying this equation when $X$ is equal to the force, $F$, we get,

$$
\frac{1}{k_{B} T}=\frac{\left\langle\nabla^{2} V_{N}\right\rangle}{\left\langle|\mathbf{F}|^{2}\right\rangle}
$$

which is connected to,

$$
\frac{1}{k_{B} T_{\operatorname{con} F}}=-\frac{\left\langle\sum_{i=1}^{N} \nabla_{i} \cdot \mathbf{F}_{i}\right\rangle}{\left\langle\sum_{i=1}^{N} \mathbf{F}_{i}^{2}\right\rangle}
$$

where, $\nabla_{i}=\left(\partial / \partial x_{i}, \partial / \partial y_{i}, \partial / \partial z_{i}\right)$ and $x_{i}, y_{i}$ and $z_{i}$ are the cartesian coordinates of particle $i$. Equation (6) has been used in computer simulations in the canonical ensemble [33], and in non-equilibrium simulations of fluids under thermal gradients $[34,35]$. Other definitions of the configurational temperature have also been proposed [33],

$$
\frac{1}{k_{B} T_{\operatorname{con} 1}}=-\left\langle\frac{\sum_{i=1}^{N} \nabla_{i} \cdot \mathbf{F}_{i}}{\sum_{i=1}^{N} \mathbf{F}_{i}^{2}}\right\rangle,
$$

which should be exact in the thermodynamic limit. However, equation (7) features a strong dependence $\mathcal{O}(1 / N)$ with system size.

Although configurational temperatures, e.g. equation (5), have been known for some time, the interest in using these temperatures in simulations was triggered by Rugh's work [36]. The configurational temperatures have later been used in experimental studies concerned with colloids under confinement since they provide an approach to test colloidsurface interactions [37].

Figure 3 shows temperature profiles for a Lennard-Jones fluid at high density (average density $\rho=0.7$ in reduced units) obtained from NEMD simulations. Clearly, the configurational (conF) and kinetic (kin) routes are fully consistent with non-equilibrium conditions, while approximate definitions of the configurational temperature (eqn. (7)) result in large deviations [35]. This discrepancy is connected to the large dependence of some of these equations with system size. This dependence becomes very important when the sampling involves small volumes (and few particles), such as the ones used in NEMD simulations to calculate local properties (see Figure 2). Figure 3 also shows the thermal conductivity extracted from the temperature profiles and Fourier's Law. The thermal conductivity is computed in this case on a local basis, using values of local thermal gradients. The agreement between the configurational and kinetic route (letting aside the approximate configurational equation, which shows again large deviations) is excellent. The comparison with equilibrium data obtained from the Green-Kubo (GK) (NVE ensemble) relation provides also another test of the NEMD approach to predict equilibrium properties by sampling local volumes. Indeed both GK and NEMD results are in excellent agreement, showing that the notion of local properties is also supported by equilibrium and non-equilibrium computations of transport properties.

\section{COUPLING EFFECTS AND INTERNAL VARIABLES}

Heat transport in fluids of anisotropic particles features an interesting physical behavior, which arises from the coupling of internal degrees of freedom, such as the molecular orientation and heat fluxes. We considered such coupling effects in fluids consisting of anisotropic dumbbells with asymmetry in 

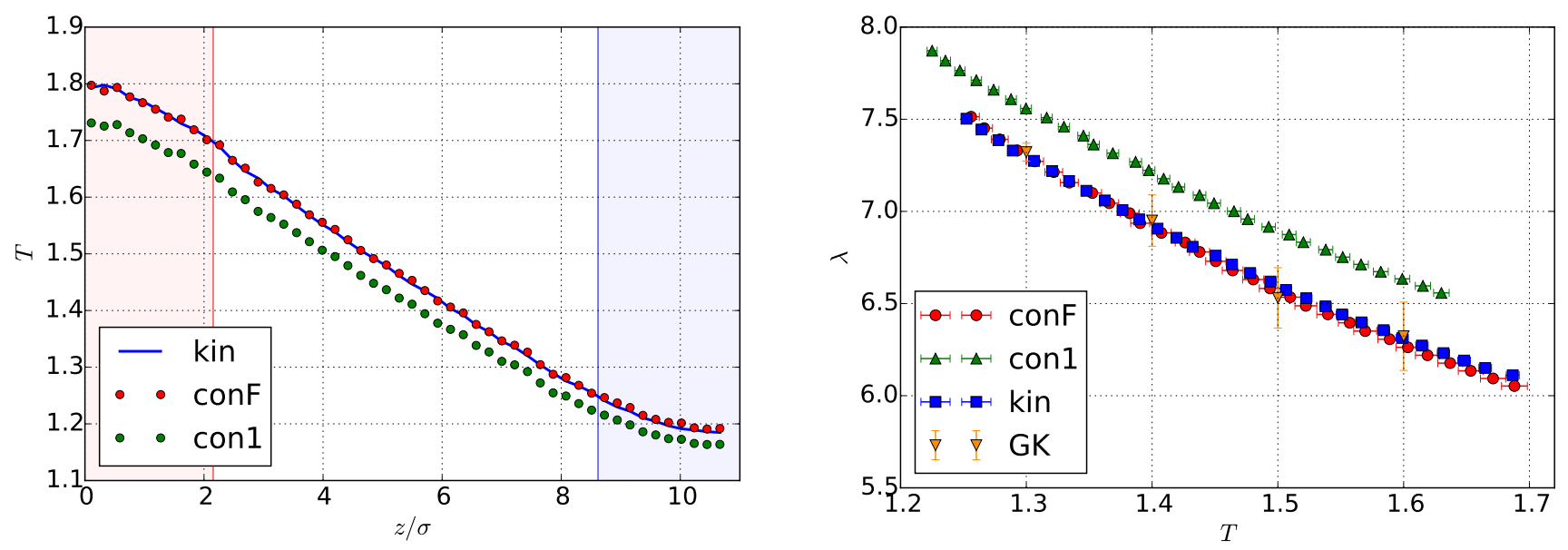

Fig. 3. (Left) Temperature profiles calculated with equations (2), (6) and (7). The red (hot) and blue (cold) areas represent the position of the thermostats. (Right) Thermal conductivity calculated from Fourier's law using local thermal gradients from the profiles represented on the left. GK refers to thermal conductivities obtained from the Green-Kubo equation. The Lennard-Jones potential cutoff is $5 \sigma$, where $\sigma$ is the atom diameter and the simulations were performed with systems consisting of 8000 particles. Data taken from reference [35]

size and/or mass [38]. The coupling of the internal degrees of freedom with the heat flux has interesting consequences. The main one is the possibility of inducing a preferred orientation in the fluid using a thermal gradient. This physical effect can be justified using Non-Equilibrium Thermodynamics. The energy needed to maintain a given orientation, defined by the vector $\mathbf{n}$ (see Figure 4), in a molecule is $E=k\langle\mathbf{n}\rangle^{2} / 2$, where $k$ is a force constant. Using this energy it is possible to derive the linear flux-force relations that describe the non-equilibrium behavior of the molecular fluid,

$$
\begin{aligned}
\frac{\partial\langle\mathbf{n}\rangle}{\partial t} & =-\frac{L_{n n}}{T} k\langle\mathbf{n}\rangle-\frac{L_{n q}}{T^{2}} \nabla T \\
\mathbf{J}_{q} & =-\frac{L_{q n}}{T} k\langle\mathbf{n}\rangle-\frac{L_{q q}}{T^{2}} \nabla T
\end{aligned}
$$

where $L_{\alpha \beta}$ are the phenomenological coefficients and $L_{n q}=$ $L_{q n}$ fulfill the Onsager reciprocal relations. Solving these equation for the stationary state, $\partial\langle\mathbf{n}\rangle / \partial t=0$, we get [38],

$$
\langle\mathbf{n}\rangle=-C \frac{\nabla T}{T}
$$

where $C=L_{n q} /\left(k L_{n n}\right)$. Equation (10) predicts a linear dependence of the orientation with the thermal gradient. Also, the orientation is predicted to vary with the inverse of the temperature. As we will see below this dependence does not always result in a weaker orientation as the temperature increases. In fact, the proximity of the fluid to a critical point can reverse such dependence, and the response can increase significantly even at high temperatures.
Figure 4 shows NEMD simulation results that illustrate the thermal orientation effect in a system consisting of dumbbell particles where the two sites have the same mass but different size, namely, $\sigma_{22} / \sigma_{11}=1 / 2$. This molecular fluid features the thermal orientation effect, consistent with the Non Equilibrium Thermodynamics predictions. The thermal orientation increases with the strength of the thermal gradient, and it does so (see ref. [38]) linearly, as predicted by equation (10). The orientation depends significantly on the thermodynamic conditions. For this model, the orientation becomes stronger at higher/lower packing fractions/temperatures.

One consequence of the thermal orientation coupling is the modification of the thermal conductivity of the fluid. According to Non-Equilibrium Thermodynamics theory,

$$
\lambda=\frac{1}{T^{2}}\left(L_{q q}-\frac{L_{n q} L_{q n}}{L_{n n}}\right),
$$

hence the thermal conductivity is determined by three phenomenological coefficients, $L_{q q}, L_{n q}=L_{q n}$ and $L_{n n}$. Hence, a computation via the Green-Kubo method will require the calculation of three independent correlation functions. From this perspective, NEMD offers a more practical approach to computing thermal conductivities, since this property can be obtained for a range of thermodynamic states using a "single" simulation by exploiting the local character of the transport properties (see Fig. 3).

The coupling effects, between molecular internal degrees of freedom and heat fluxes, can be extended to situations where more than one external field, e.g. thermal and gravitational, is applied to the system of interest [39]. This idea has 


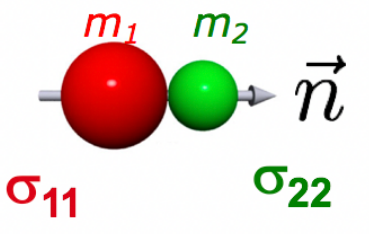

$U_{i j}=4 \varepsilon\left(\frac{\sigma_{i j}}{r_{i j}}\right)^{12} \varepsilon_{1}=\varepsilon_{2}$

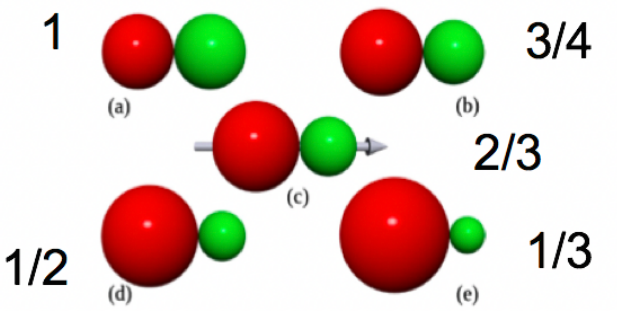

temperature $T^{*}$

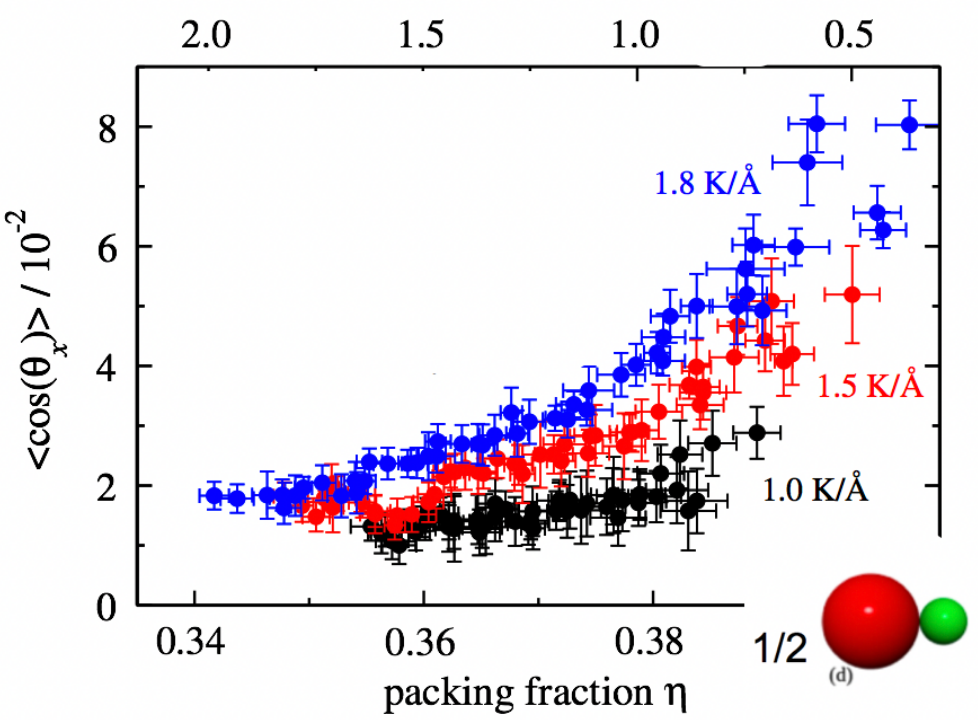

Fig. 4. (Left) Sketch of dumbbells consisting of tangent spheres of different size or mass $\left(\mathrm{m}_{1}, \mathrm{~m}_{2}\right)$. The fractions indicate the size ratios, and the equation represents the pair interaction between the dumbbell sites. The arrow denotes the vector $\vec{n}$ that defines the orientation of the molecules. (Right) Dependence of the orientation induced by the thermal gradient with packing fraction/temperature. The results correspond to dumbbells with size ratio 1/2 (see snapshot superimposed on the figure) and data for three thermal gradients are shown. Figures adapted from reference [38]

been used to disentangle the contributions of temperature and density gradients to the overall fluid thermal orientation.

Coupling of internal degrees of freedom with heat fluxes is of particular interest in water. The coupling results in the polarization of the fluid, and consequently, in a thermally induced electrostatic field. Using the Non-Equilibrium Thermodynamics theory in a similar way as shown above for dumbbell fluids, it is possible to derive an equation that connects the electrostatic field, E, to the thermal gradient [40],

$$
\mathbf{E}=\left(1-\frac{1}{\varepsilon_{r}}\right) \frac{L_{p q}}{L_{p p}} \frac{\nabla T}{T}=S_{T P} \nabla T
$$

where $L_{\alpha \beta}$ represents again the phenomenological coefficients, $\varepsilon_{r}$, is the permittivity of the solvent and $S_{T P}$ is the Thermo-polarization coefficient. Equation (12) is remarkably similar to the equation defining thermoelectric effects, and $S_{T P}$ in equation (12) would play an equivalent role as the Seebeck coefficient [28].

NEMD simulations offer an efficient approach to quantify the coefficient $S_{T P}$ and therefore the strength of the polarization effect. The dependence of the magnitude and sign of this coefficient with temperature and density is fairly complex. It has been observed that the coefficient changes sign with temperature [27]. The sign of the coefficient is defined by a balance of dipolar and quadrupolar terms, which contribute to the electrostatic field of water. The quadrupolar term depends strongly on the thermal expansion and dominates the polarization response of water at high temperatures.

The thermally induced polarization increases significantly upon approaching a critical point. This effect is connected to the large increase of the thermal expansion near criticality conditions and the eventual divergence of this property. The increase of the polarization response along the critical isochore can be described well with the equation, $\left|S_{T P}\right|=\left(1-T_{c} / T\right)^{-\gamma}$. This equation follows the same temperature dependence observed in other response properties (thermal expansion or isothermal compressibility) of water as the temperature approaches the critical point [22]. The exponent $\gamma=1.239$, corresponds to the universality class of Ising-like systems, such as water, and describes well the increase of the thermo-polarization coefficient near critical conditions (see inset in Figure 5).

\section{CONCLUSIONS AND FINAL REMARKS}

Non-Equilibrium Molecular Dynamics (NEMD) simulations offer a powerful approach to investigate the nonequilibrium response of molecular fluids to thermal gradients. NEMD simulations have been used to test the validity of the local equilibrium hypothesis. It is found that a wide range of systems follow a linear response, and it is possible 

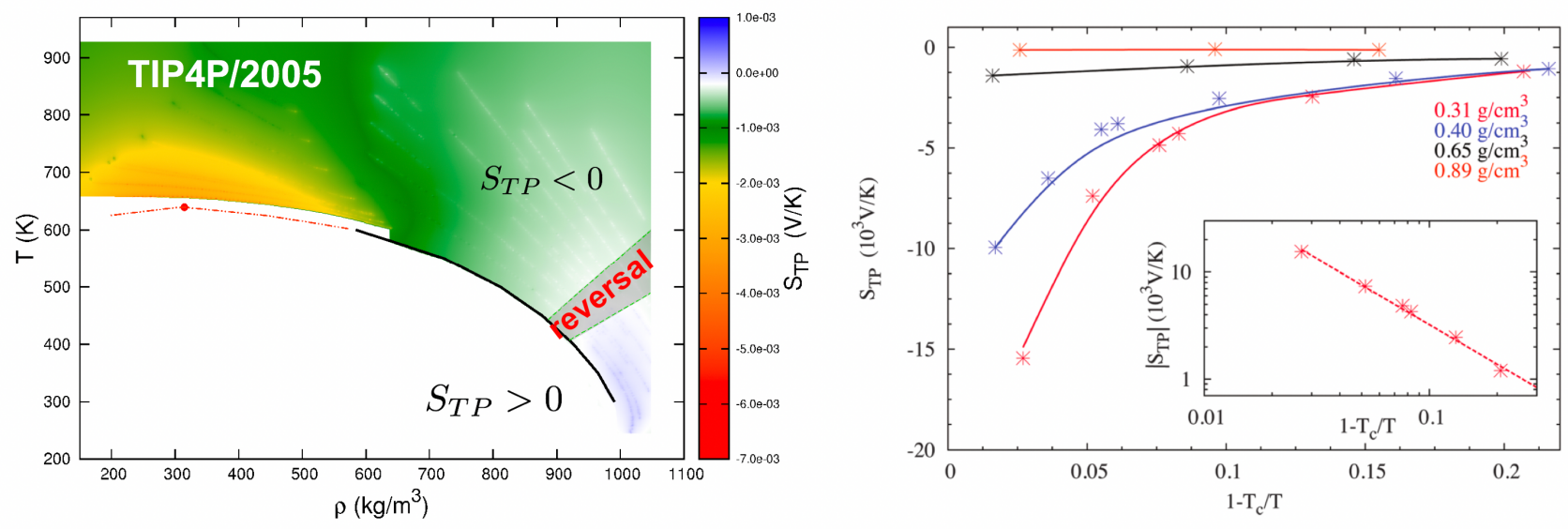

Fig. 5. (Left) Variation of the thermo-polarization (TP) coefficient in TIP4P-2005 water as a function of temperature and density. The white region corresponds to coexistence conditions. The full and dashed lines represent the coexistence line, and the point is an estimate of the critical point for this water model. The "reversal" region indicates the area of the temperature/density plane where the TP coefficient changes sign. The color code shows the magnitude of the TP effect, see color code in the figure. (Right) Variation of the TP coefficient along different isochores, including the critical one $(\rho=0.31 \mathrm{~g} / \mathrm{cc})$. The inset shows the dependence of the coefficient (in absolute value) with the distance to the critical point, $1-T_{c} / T$. The slope of the line is 1.239. Figures reproduced from reference [22]

to describe their local behavior using equilibrium properties. This fact brings some benefits, as it is possible to quantify efficiently thermophysical properties (equations of state, thermal conductivities) using a single NEMD simulation. NEMD simulations under thermal gradients rely on the computation of local temperatures. Numerical simulations have shown good agreement between kinetic and configurational temperatures, and support the use of the kinetic definition to calculate temperatures in standard NEMD simulations.

The coupling of internal degrees of freedom and heat fluxes gives rise to interesting non-equilibrium effects, such as molecular orientation of non-polar fluids and water. The coupling of internal degrees of freedom and heat fluxes raises some questions regarding the use of standard Green-Kubo equations in molecular fluids, in particular, the need to include additional contributions in the Green-Kubo computations, in order to take into account the coupling effects. Further work in this direction would be desirable.

Future developments in the thermal polarization of water may focus on NEMD simulations near critical conditions, where there is now evidence supporting the possibility of inducing significant polarization responses using modest thermal gradients. Indeed, NEMD simulations indicate that thermal gradients of $\sim 10^{6-8} \mathrm{~K} / \mathrm{m}$, which can be generated easily at the nanoscale using metallic nanoparticles, could induce electrostatic fields of $\sim 10^{4-6} \mathrm{~V} / \mathrm{m}$. Such predictions await experimental verification. Light scattering techniques [41] could be helpful to advance experimental studies of these fascinating non-equilibrium phenomena.

\section{Acknowledgement}

I would like to thank Jeff Armstrong, Dick Bedeaux, Chris Daub, Irene Iriarte-Carretero, Miguel Angel Gonzalez, Signe Kjelstrup, Anders Lervik, Frank Römer, Miguel Rubí and Jan Sengers for many discussions over the years and for fruitful collaborations. I thank the EPSRC (EP/J003859/1) and the Research Council of Norway (Project 221675) for financial support and the Imperial College High-Performance Computing Service for providing computational resources. Finally, I would like to thank William Hoover for the inspiration in the field of non-equilibrium simulations.

\section{References}

[1] C. Ludwig, Sitz. ber. Akad. Wiss. Wien Math.-Nat. wiss. Kl 50, 539 (1856).

[2] C. Soret, Etat d'équilibre des dissolutions dont deux parties sont portées à des températures différentes, Arch. Sci. Phys. Nat., Geneve 2, 48 (1879).

[3] S. Wiegand, Thermal diffusion in liquid mixtures and polymer solutions, J. Phys.: Condens. Matter 16, R357 (2004).

[4] R. Piazza, A. Parola, Thermophoresis in colloidal suspensions, J. Phys.: Condens. Matter 20, 153102 (2008).

[5] S. Putnam, D. Cahill, G. Wong, Temperature Dependence of Thermodiffusion in Aqueous Suspensions of Charged Nanoparticles, Langmuir 23, 9221 (2007).

[6] M. Reichl, M. Herzog, A. Götz, D. Braun, Temperature Dependence of Thermodiffusion in Aqueous Suspensions of Charged Nanoparticles, Langmuir 23, 9221 (2007).

[7] T. Kirkpatrick, J.O. de Zarate, J.V. Sengers, Nonequilibrium Casimir-like Forces in Liquid Mixtures, Phys. Rev. Lett. 115, 035901 (2015). 
[8] P. Baaske, F. Weinert, S. Duhr, K. Lemke, M. Russel, D. Braun, Extreme accumulation of nucleotides in simulated hydrothermal pore systems, Proc. Natl. Acad. Sci. USA 104, 9346 (2007).

[9] S. Duhr, D. Braun, Thermophoretic Depletion Follows Boltzmann Distribution, Phys. Rev. Lett. 96, 168301 (2006).

[10] C. Debuschewitz, W. Köhler, Molecular Origin of Thermal Diffusion in Benzene + Cyclohexane Mixtures, Phys. Rev. Lett. 87, 055901 (2001).

[11] K. Eslahian, A. Majee, M. Maskos, A. Würger, Specific salt effects on thermophoresis of charged colloids, Soft Matter 10, 1931 (2014).

[12] S. Di Lecce, T. Albrecht, F. Bresme, A computational approach to calculate the heat of transport of aqueous solutions, Sci. Rep., 7, 44833 (2017).

[13] W.G. Hoover, Nonequilibrium Molecular Dynamics, Ann. Rev. Phys. Chem. 34, 103 (1983).

[14] D. J. Evans, G. P. Morris, Statistical Mechanics of Nonequilibrium Liquids, Academic Press, New York, 1990.

[15] F. Bresme, A. Lervik, J. Armstrong, Non-equilibrium Molecular Dynamics, Chapter 6, in Experimental Thermodynamics Volume X: Non-Equilibrium Thermodynamics with Applications, D. Bedeaux, S. Kjelstrup, J.V. Sengers eds., IUPAC, p. 105 (2016).

[16] D.J. Evans, Homogeneous NEMD algorithm for thermal conductivity-Application of non-canonical linear response theory, Phys. Lett., 91A, 457 (1982).

[17] D.P. Hansen, D.J. Evans, A generalized heat flow algorithm, Mol. Phys. 81, 767 (1994).

[18] J. Armstrong, F. Bresme, Water polarization induced by thermal gradients: The extended simple point charge model (SPC/E), J. Chem. Phys. 139, 014504 (2013).

[19] A. Tenenbaum, Local equilibrium in stationary states by molecular dynamics, Phys. Rev. A 28, 3132 (1983).

[20] B. Hafskjold, in Thermal Nonequilibrium Phenomena in Fluid Mixtures, Lecture Notes in Physics, ed. W. Köhler, S. Wiegand, Springer, p. 3, (2001).

[21] F. Müller-Plathe, A simple nonequilibrium molecular dynamics method for calculating the thermal conductivity, J. Chem. Phys. 106, 6082 (1997).

[22] I. Iriarte-Carretero, M.A. Gonzalez, J. Armstrong, F. Fernandez-Alonso, F. Bresme, The rich phase behavior of the thermopolarization of water: from a reversal in the polarization, to enhancement near criticality conditions, Phys. Chem. Chem. Phys. 18, 19894 (2016).

[23] G. Bussi, D. Donadio, M. Parrinello, Canonical sampling through velocity rescaling, J. Chem. Phys. 126, 014101 (2007).

[24] J. H. Irving, J. G. Kirkwood, The Statistical Mechanical Theory of Transport Processes. IV. The Equations of Hydrodynamics, J. Chem. Phys. 18, 817 (1950).
[25] B.D. Todd, D.J. Evans, P.J. Daivis, Pressure tensor for inhomogeneous fluids, Phys. Rev. E, 52, 1627 (1995).

[26] A.S. Tascini, J. Armstrong, E. Chiavazzo, M. Fasano, P. Asinari, F. Bresme, Thermal transport across nanoparticle-fluid interfaces: the interplay of interfacial curvature and nanoparticle-fluid interactions, Phys. Chem. Chem. Phys. 19, 3244 (2017).

[27] J. Armstrong, F. Bresme, Temperature inversion of the thermal polarization of water, Phys. Rev. E 92, 060103 (2015).

[28] S.R. de Groot, P. Mazur P., Non-equilibrium thermodynamics, New York, Dover, (1984).

[29] F. Römer, A. Lervik, F. Bresme, Nonequilibrium molecular dynamics simulations of the thermal conductivity of water: A systematic investigation of the SPC/E and TIP4P/2005 models, J. Chem. Phys. 137, 074503 (2012).

[30] J. Casas-Vázquez J, D. Jou, Temperature in non-equilibrium states: a review of open problems and current proposals, Rep. Prog. Phys. 66, 1937 (2003).

[31] L.D. Landau, E.M. Lifschitz, Statistical physics, part 1. Oxford: Pergamon, (1969).

[32] J.P. Hansen, I.R. Macdonald, Theory of simple liquids, $3^{\text {rd }}$ ed. Amsterdam: Academic Press (2013).

[33] O. Jepps, G. Ayton, D. Evans, Microscopic expressions for the thermodynamic temperature, Phys. Rev. E. 62, 4757 (2000).

[34] A. Lervik A, O. Wilhelmsen, T.T. Trinh, H.R. Nagel, Finitesize and truncation effects for microscopic expressions for the temperature at equilibrium and nonequilibrium, J. Chem. Phys. 143, 114106 (2015).

[35] N. Jackson, J. M. Rubi, F. Bresme, Non-equilibrium molecular dynamics simulations of the thermal transport properties of Lennard-Jones fluids using configurational temperatures, Mol. Sim. 42, 1214 (2016).

[36] H.H. Rugh, Dynamical Approach to Temperature, Phys. Rev. Lett. 78, 772 (1997).

[37] D.G. Grier, Y. Han, Anomalous interactions in confined charge-stabilized colloid, J. Phys.: Condens. Matter. 16, S4145 (2004).

[38] F. Römer, F. Bresme, J. Muscatello, D. Bedeaux, J.M. Rubi, Thermomolecular Orientation of Nonpolar Fluids, Phys. Rev. Lett. 108, 105901 (2012).

[39] C.D. Daub, J. Tafjord, S. Kjesltrup, D. Bedeaux, F. Bresme, Molecular alignment in molecular fluids induced by coupling between density and thermal gradients, Phys. Chem. Chem. Phys. 18, 12213 (2016).

[40] F. Bresme, A. Lervik, D. Bedeaux, S. Kjelstrup, Water Polarization under Thermal Gradients, Phys. Rev. Lett. 101, 020602 (2008).

[41] J.V. Sengers, private communication (2016). 


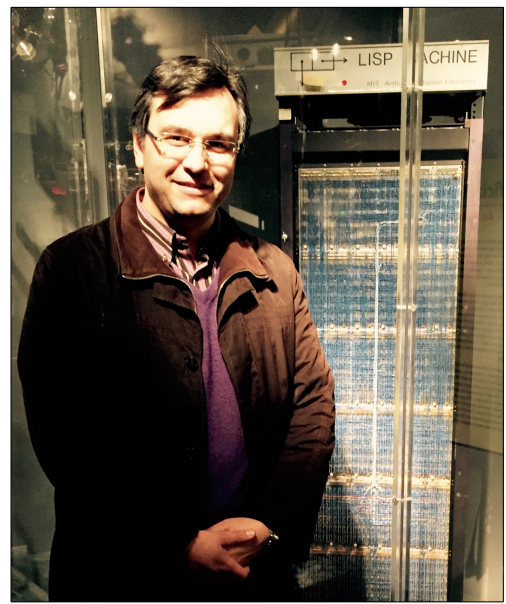

Fernando Bresme (FB) is Professor of Chemical Physics in the Department of Chemistry at Imperial College London, where he heads the Computational Chemical Physics group. His research is concerned with the computational and theoretical investigation of equilibrium and non-equilibrium response of soft matter systems. He and his group are working actively in the investigation of thermal transport processes in soft materials.

FB is recipient of the McBain medal in Colloid and Interface Science and he has been awarded an EPSRC Leadership Fellowship. He is a member of the Statistical Mechanics and Thermodynamics group of the Royal Society of Chemistry and of the Faraday Division Council, and Adjunct Professor of Computational Chemical Physics at the Norwegian University of Science and Technology. FB has co-organized the Non-Equilibrium Simulation School (NESS) and the Non-Equilibrium Simulation Conference (NESC), and he is co-chair of the Non-Equilibrium Thermodynamics session of the Symposium on Thermophysical properties held at the University of Boulder (USA). 